\title{
Telepractice in service delivery: A survey of perspectives and practices of speech and language therapists in Ireland during COVID-19
}

\author{
Eilis Farren*, Duana Quigley and Yvonne Lynch \\ Department of Clinical Speech and Language Studies, Trinity College Dublin, Ireland
}

Received 19 August 2021

Accepted 20 December 2021

\begin{abstract}
.
BACKGROUND: COVID-19 accelerated telepractice implementation in speech and language therapy (SLT) in Ireland. OBJECTIVE: This study documents the service delivery changes that took place in the SLT profession in Ireland during the public health crisis.

METHODS: An online survey of speech and language therapists (SLTs) in Ireland was conducted from June-September 2020 to investigate their perceptions of telepractice. Data were analysed using descriptive and inferential statistics and frequency distribution.

RESULTS: 173 SLT responses were analysed. Over half of the participants worked in urban locations. Respondents' years of experience varied from less than four years to over 20 years. Slightly over half the participants reported using telepractice, with $85 \%$ starting to use telepractice in the six months prior to the survey. Telepractice uptake was not influenced by participants' professional experience or geographical location $(p>0.05)$. Almost all participants who used telepractice were trained informally (92\%). Telepractice was most commonly used with school-aged children with developmental language and speech sound disorders. Respondents perceived that telepractice was not suitable for all individuals who need SLT, including those with complex needs. Clinicians reported that telepractice facilitated access to therapy for clients and opportunities to see clients in their own environments. Technology barriers were the biggest hurdle to telepractice use.

CONCLUSIONS: Uptake of telepractice by the SLT profession in Ireland was widespread during COVID-19, highlighting the profession's flexibility and innovation. Respondents indicated they are likely to continue to use telepractice as a complementary service delivery model post-COVID due to the distinct benefits for clinicians and clients.
\end{abstract}

Keywords: Telehealth, telepractice, speech and language therapy, service delivery

\section{Background}

Telehealth refers to the delivery of health and medical services via telecommunication technologies

${ }^{*}$ Corresponding author: Eilis Farren, Department of Clinical Speech and Language Studies, Trinity College Dublin, 7-9 South Leinster Street, Dublin 2, Ireland. E-mail: eilisfarren@gmail.com. when clients and clinicians are separated by distance (ASHA, 2021). Telepractice has been adopted as a term for the delivery of speech and language therapy (SLT) services via information and communications technology (ICT) (AHA, 2021). While telehealth and telepractice may be used interchangeably, telehealth may suggest that SLT services are only delivered in 
healthcare settings when in reality, services may be provided virtually in a range of social, health and educational settings. Telepractice will be the term used in this article to describe SLT services delivered via ICT.

There is a growing evidence base to support telepractice in many areas of SLT, with children and adults with diverse speech, language, communication and swallowing disorders. Studies investigating telepractice use with individuals with autism spectrum disorder, attention deficit hyperactivity disorder and anxiety disorders reported a range of therapeutic gains, including increased caregiver confidence in supporting social communication and reductions in emotional dysfunction (Mac Evilly \& Brosnan, 2020). School-age children made progress following telepractice delivery of the Van Riper approach to articulation intervention and achieved goals based on their individual education plan (IEP) following a combined speech and language intervention programme using telepractice (Wales, Skinner \& Hayman, 2017). A randomised control trial investigating the feasibility of telepractice-delivery of dysarthria intervention for children with cerebral palsy reported improvements in speech intelligibility and communicative independence (Pennington et al., 2019). Nordio et al. (2018) conducted a systematic review and found that telepractice improved adherence to treatment recommendations in patients with dysphagia. Outcomes of voice therapy delivered via telepractice were reported to be comparable to face-to-face therapy for clients with voice and upper airway disorders in the treatment of vocal hyperfunction, vocal nodules, vocal fold paralysis and oedema (Doll, Braden \& Thibeault, 2020). Telepractice was used effectively to target and monitor motor speech function in individuals with Parkinson's disease (Swales et al., 2019) amongst a range of other conditions. Collectively, the literature suggests that telepractice is as effective as face-to-face delivery for many forms of SLT.

Telepractice may offer additional benefits for both clinicians and clients. Telepractice can reduce access barriers associated with attending face-to-face services, allowing clients to engage with services from a greater distance, regardless of transport, mobility or childcare. For clinicians, it may facilitate effective caseload management, with reductions in time spent travelling or booking, preparing and cleaning clinic rooms, clinicians may have more available time to connect with clients (Tucker, 2012). Moreover, there are favourable outcomes associated with service delivery to clients in their functional environments (Grillo, 2017; WHO, 2001), which can be achieved using telepractice. High levels of client- and clinician-reported satisfaction with telepractice have been documented in several studies (Morris et al., 2019; Swales et al., 2019).

In order to access the benefits of telehealth, specific ICT equipment and competencies are required. Low levels of ICT literacy, on behalf of the client or the clinician, have been reported as one of the greatest hurdles in the successful adoption of telepractice in SLT (Morris et al., 2019). Clinicians have also indicated that telepractice is not feasible if videoconferencing equipment and broadband connection are not available to clients (Greenhalgh et al., 2020). Greater adoption of telepractice may contribute to a deepening of the digital divide, which describes the positive correlation between poorer health status and lack of access to health information and the internet (Hernandez \& Roberts, 2018). Often the risk of digital marginalisation may be mitigated by increasing investments in ICT infrastructure and improving accessibility of telepractice devices, connectivity and education for clients (WHO, 2020).

Recently published policies have identified these challenges and propose a number of steps to facilitate greater implementation of telepractice. For example, in 2020, the World Health Organisation (WHO) released a global strategy for the digital transformation of healthcare, which recognises the role of telepractice in the delivery of efficient, cost-effective and person-centred care in line with the Sustainable Development Goals. The Irish Department of Health (2019) have also identified telepractice as a key area for development in the modernisation of Ireland's healthcare services. Ireland's national healthcare policy, Sláintecare (2019) outlines plans to strengthen and expand this service delivery model by 2028 (Department of Health, 2019). The Irish Association of Speech and Language Therapists (IASLT) also supports telepractice use, provided its delivery is based on evidence-based care and the quality of the service is at least equivalent to in-person clinical care (IASLT, 2020).

In spite of these strategic policy priorities and the growing evidence base of its benefits, the implementation of telepractice in SLT in Ireland was limited prior to the COVID-19 pandemic. This low uptake may relate to the novelty of telepractice as a service delivery model within the profession (Kuva, Mati \& Dokoza, 2020). While little is known about telepractice use amongst speech and language therapists 
(SLTs) in Ireland, the literature suggests that uptake of telepractice is varied around the world. Australia, a recognised leader in telepractice, reported high rates of telepractice use. In a survey conducted in 2012 (Hill \& Miller), the majority of Australian SLTs reported using telepractice as a service delivery model, although most reported that telepractice was new to them in the last 6 years $(80.6 \%)$. In the USA, telepractice adoption is growing steadily with an ASHA survey reporting that $64 \%$ of 476 respondents used telepractice to deliver SLT (ASHA, 2016). However, implementation of telepractice has been slower in other countries. For example, in India a survey which recruited SLTs and audiologists reported 12\% of 205 participants used telepractice (Mohan et al., 2017). More recently, an online survey completed in Hong Kong found that just over one third (38\%) of participating SLTs used telepractice, but this was predominantly in response to the COVID19 pandemic (Fong, Tsai \& Yiu, 2020). Similarly, in a survey investigating telepractice use amongst SLTs in response to COVID-19 in Croatia, $74 \%$ of participants used telepractice at the time of survey completion (Kuva, Mati \& Dokoza, 2020). Across healthcare professions in Ireland, the introduction and development of telepractice was in its infancy until 2019, with an estimated 3\% of healthcare workers using video consultations with clients before the outbreak of COVID-19 (HSCP, 2020). Following a search of the literature, no studies were identified which surveyed the practices and perspectives of SLTs in relation to telepractice within the Irish context.

Based on previous international studies, adopting telepractice is likely to be influenced by SLTs' perceptions of this service delivery model as well as the barriers and facilitators of telepractice in different contexts (Swales et al., 2019). With clinician satisfaction acting as a fundamental factor in the acceptance and use of telepractice (Morris et al., 2019), it is important to understand the diverse perspectives of clinicians to facilitate the embedding of telepractice as a complementary service delivery model. Internationally there is consensus that the limited training, clinical practice standards and support with telepractice are exacerbating scepticism amongst SLTs, inhibiting uptake (Fong, Tsai \& Yiu, 2020; Smith et al., 2020; Swales et al., 2019; Zughni et al., 2020). Low levels of ICT literacy have been reported as one of the greatest hurdles in the successful adoption of telepractice in SLT (Greenhalgh et al., 2020; HSCP, 2020; Morris et al., 2019;
Zughni et al., 2020) along with logistical considerations, including the lack of physical proximity to the client and over-reliance on the client's parent or carer in telepractice sessions (Akamoglu et al., 2018). Tucker (2012) conducted a web-based survey of SLTs working in schools in America and identified that the dearth of evidence surrounding treatment efficacy and validity of assessments delivered using telepractice were significantly impeding its implementation.

The COVID-19 pandemic has been a major disruptor of services and has seen the profession of SLT respond with innovation and creativity to maintain service delivery. Many SLTs turned to telepractice to enable them to continue to deliver services in the context of public health restrictions. Practitioners have reported that telepractice is a flexible service delivery option which facilitates safe continuity of care during the COVID-19 pandemic (Dimer et al., 2020; Tohidast et al., 2020). The present study documents the change in service delivery models in Ireland during the COVID-19 pandemic through telepractice implementation and suggests how this innovation could be embedded into long term service delivery to ensure effective and sustainable client-centred care.

The study aim was to examine how SLTs in Ireland viewed telepractice and their use of telepractice during the COVID-19 pandemic. Several research questions were devised to address this aim:

1. What were the patterns of use of telepractice by SLTs working in Ireland?

2. What are the views of Irish SLTs regarding telepractice as a service delivery model?

3. What barriers and facilitators to using telepractice are reported by SLTs working in Ireland?

4. What are SLTs' views on the supports needed for effective use of telepractice?

The authors hypothesised that telepractice had been a major change to typical SLT service delivery and that there were mixed perspectives of the advantages and disadvantages of its rapid implementation.

\section{Methods}

\subsection{Study design}

A cross-sectional online survey method was chosen as the study design to enable anonymous, efficient and cost-effective collection of data. To help ensure robust quality standards, the design of this study was guided by the Consensus-Based Checklist for 
Reporting of Survey Studies (CROSS) (Sharma et al., 2021) (see appendix 1). Prior to commencing the study, research ethics approval was obtained from the Research Ethics Committee of the School of Linguistic, Speech and Communication Sciences, Trinity College Dublin, approval number TT38.

\subsection{Survey development}

An anonymous online survey was developed specifically for the current study. It was structured to address the study's research questions in relation to SLTs' work practices and perspectives about telepractice as a service delivery model. This crosssectional survey collected participant demographics, telepractice experience, perspectives on the advantages and disadvantages of telepractice and supports needed for implementing telepractice services. One SLT piloted the survey and provided constructive feedback, which was used to revise and refine the survey tool before distribution. Survey questions were designed to be easy to follow and completed in less than 15 minutes (see appendix 2 for survey questions). The survey was constructed in the Qualtrics platform (Qualtrics Provo, 2005). Qualtrics provided a GDPR compliant way to deliver the survey anonymously and remotely online.

\subsection{Survey dissemination}

Individuals registered with the Irish regulatory body for SLTs (CORU) with experience working as an SLT in Ireland were invited to participate. The survey was open to SLTs with and without experience using telepractice. Screening questions were used to exclude respondents who did not meet the inclusion criteria. The anonymous online survey was circulated via Twitter and email. (i.e., twitter account of the IASLT, twitter account of the university of the authors, and SLT professional email distribution list). Social media adverts provided an online link to the anonymous survey where potential participants could review the participant information leaflet before deciding whether to continue. Participation was voluntary and the survey was distributed online in June 2020, remaining open for 13 weeks until September 2020.

\subsection{Data analyses}

Results of the current study were analysed using SPSS Statistics version 26 (IBM, 2019). Analy- ses included chi-square tests of independence and comparisons of contingency tables to determine whether the relationship between telepractice use and participants' demographic details was statistically significant and to quantitatively analyse the correlations between these variables. Frequency distribution was used to investigate associations between results that were not statistically significant.

\section{Results}

The results presented below detail the patterns of telepractice use of respondents in Ireland and the perceived benefits, barriers and facilitators of using this service delivery model.

\subsection{Survey respondents}

In total, 189 responses were received. Five responses were excluded as the respondents indicated they did not meet the inclusion criteria, and a further 11 responses were excluded as they completed less than $40 \%$ of the survey. The results are based on the 173 completed responses received. Due to the sampling method used, a response rate could not be calculated for this study. This sample represents approximately $9 \%$ of SLTs registered to work in Ireland in 2020 (CORU Registration Statistics, 2020) and can be considered representative at a $90 \%$ confidence level with a $6 \%$ margin of error (Qualtrics, 2021). Demographic data related to all respondents are shown in Table 1.

Participants had a range of experience levels working as SLTs, and the majority were working in client supporting roles at basic or senior grade level (83\%, $n=144)$. Over two-thirds reported working in community or disability settings $(70 \%, n=121)$. Over half of the respondents worked in urban locations, with the remainder indicating they worked in rural or other locations (see Table 1).

\subsection{Telepractice use}

Survey respondents were evenly distributed in their telepractice experience. Slightly over half of the participants reported they used telepractice $(51 \%$, $n=89)$, while $49 \%$ did not $(n=84)$ (see Table 1$)$. The majority of respondents $(85 \%, n=75)$ with telepractice experience had used it for less than 6 months at the time of survey completion (Summer 2020). For the remainder of participants, $2 \%(n=2)$ had been using telepractice for 7 months- 1 year, $6 \%(n=6)$ 
Table 1

Participant Demographics

\begin{tabular}{|c|c|c|c|}
\hline \multicolumn{2}{|l|}{ All Participants $(n=173)$} & \multicolumn{2}{|c|}{ Participants who Use Telepractice $(n=88)$} \\
\hline \multicolumn{4}{|c|}{ Years of Experience } \\
\hline$<4$ years & $35(20 \%)$ & $<4$ years & $16(18 \%)$ \\
\hline $5-10$ years & $45(26 \%)$ & $5-10$ years & $21(24 \%)$ \\
\hline $11-20$ years & $49(28 \%)$ & $11-20$ years & $24(27 \%)$ \\
\hline $20+$ years & $45(26 \%)$ & $20+$ years & $27(31 \%)$ \\
\hline \multicolumn{4}{|c|}{ Staff Grade } \\
\hline Basic grade & $64(37 \%)$ & Basic grade & $30(34 \%)$ \\
\hline Senior grade & $81(46 \%)$ & Senior grade & $44(50 \%)$ \\
\hline Clinical specialist & $9(5 \%)$ & Clinical specialist & $5(6 \%)$ \\
\hline Manager & $15(9 \%)$ & Manager & $6(7 \%)$ \\
\hline Other & $5(3 \%)$ & Other & $3(3 \%)$ \\
\hline \multicolumn{4}{|c|}{ Type of Setting } \\
\hline Acute hospital & $23(13 \%)$ & Acute hospital & $11(13 \%)$ \\
\hline Outpatient hospital & $4(2 \%)$ & Outpatient hospital & $3(3 \%)$ \\
\hline Rehabilitation hospital & $2(1 \%)$ & Rehabilitation hospital & $2(2 \%)$ \\
\hline Community services- children & $57(33 \%)$ & Community services- children & $25(29 \%)$ \\
\hline Community services- adults & $12(7 \%)$ & Community services- adults & $7(8 \%)$ \\
\hline Disability setting & $52(30 \%)$ & Disability setting & $25(29 \%)$ \\
\hline Child and adolescent mental health services & $8(5 \%)$ & Child and adolescent mental health services & $7(8 \%)$ \\
\hline Primary school & $1(1 \%)$ & Primary school & $1(1 \%)$ \\
\hline Other & $13(8 \%)$ & Other & $6(7 \%)$ \\
\hline \multicolumn{4}{|c|}{ Location of Setting } \\
\hline Urban location & $110(63 \%)$ & Urban location & $56(64 \%)$ \\
\hline Rural location & $43(25 \%)$ & Rural location & $21(24 \%)$ \\
\hline Other & $21(12 \%)$ & Other & $11(12 \%)$ \\
\hline
\end{tabular}

were using it for $2-4$ years and $7 \%(n=7)$ reported using it for more than 5 years.

Based on the statistical analysis conducted, there was no association between the likelihood of clinicians using telepractice and their staff grade, number of years qualified as an SLT, or the type or location of their work setting, at a significance level of $p=0.05$. The following calculation was used to determine this result; $\mathrm{x}^{2}(4, N=173)=1.14, p=0.05$.

Respondents indicated they were most likely to use telepractice for intervention services $(25 \%, n=69)$ and to review or monitor previously learned skills $(21 \%, n=58)$ teaching assistants and other professionals $(16 \%, n=43)$ (see Fig. 1).

Respondents indicated they used telepractice with clients across all age groups, from $0-5$ years to over 66 years (see Fig. 2). Telepractice was most often used with children and young people aged between six and eighteen years of age (37\% of respondents, $n=54)$.

The most common client groups for receiving services using telepractice were children with speech sound disorders $(14 \%, n=33)$, children with developmental language disorder (DLD) $(14 \%, n=33)$ and autism spectrum disorders $(10 \%, n=23)$, and adults with dysphagia $(11 \%, n=27)$ (see Fig. 3).

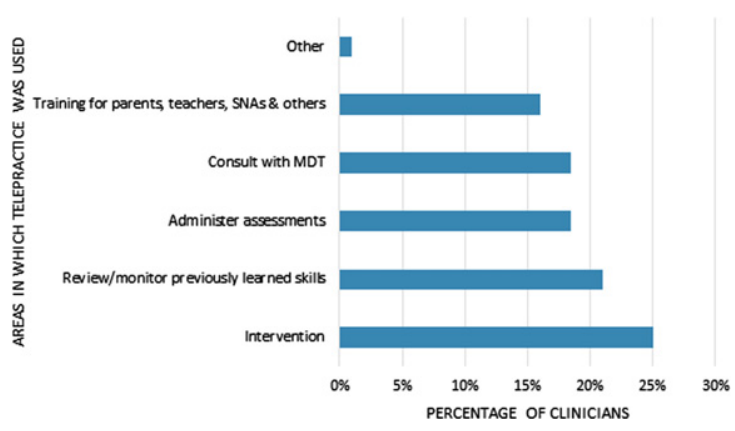

Fig. 1. Activities conducted via telepractice.

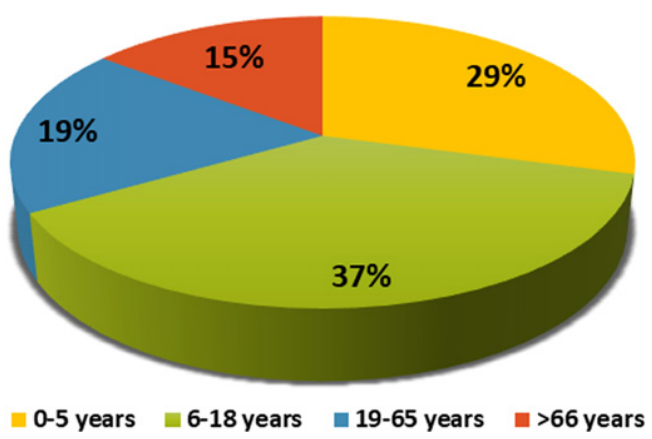

Fig. 2. Age groups of clients who received speech and language therapy through telepractice. 


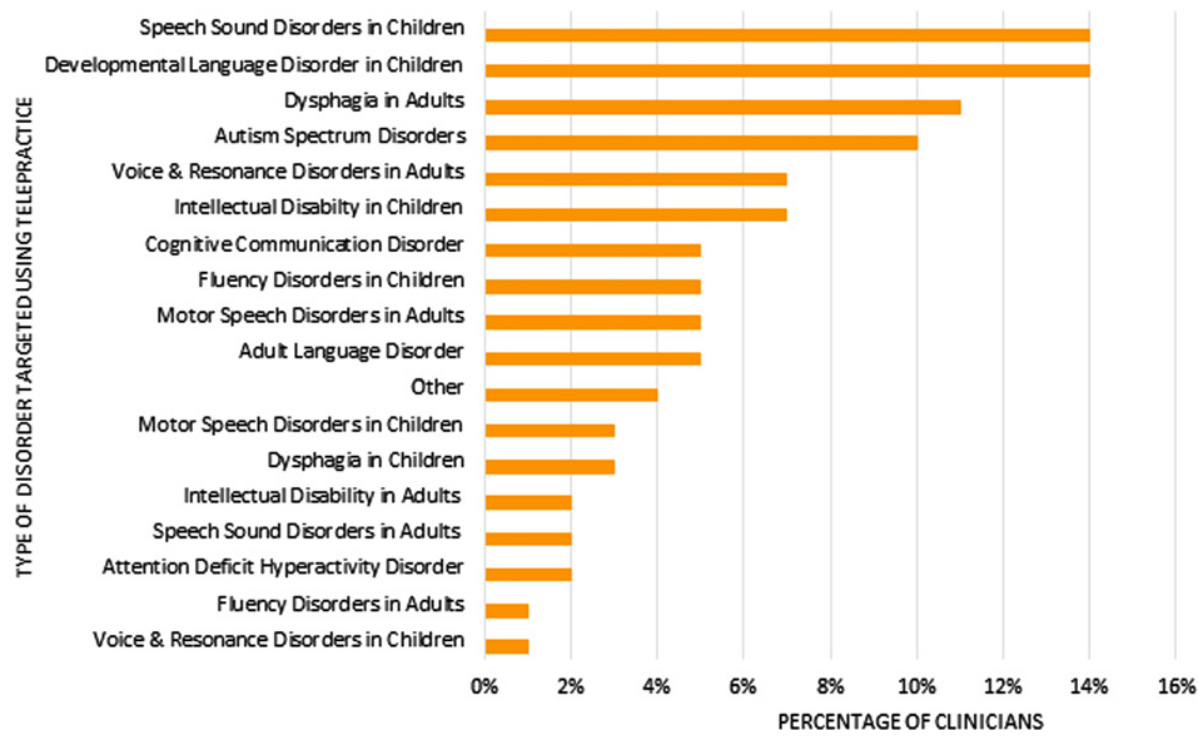

Fig. 3. Type of speech and language therapy delivered using telepractice.

Table 2

Benefits of using telepractice

\begin{tabular}{lc}
\hline Benefit & $\begin{array}{r}\text { Percentage of } \\
\text { Respondents }\end{array}$ \\
\hline Caseload management and facilitating service & $44 \%$ \\
$\quad$ continuity during the pandemic & $31 \%$ \\
Can see individual in their own setting & $9.5 \%$ \\
Decreased travel time for SLT & $8 \%$ \\
Ease of access to services for clients and families & $7.5 \%$ \\
Professional growth & $2 \%$ \\
Greater collaboration with families due to a & \\
$\quad$ more active role for parents and caregivers & $2 \%$ \\
Supporting generalisation and carryover as skills & \\
$\quad$ were already embedded in the home & \\
$\quad$ environment & $1 \%$ \\
Telepractice is a helpful monitoring/check-in & \\
$\quad$ support for face-to-face therapy & \\
\hline
\end{tabular}

\subsection{Benefits of telepractice}

Survey respondents were asked to identify what they felt was the biggest benefit of using telepractice (see Table 2). The most popular benefits identified were caseload management and service continuity. Over a third reported the ability to work with the client in their own environment $(31 \%, n=37)$ as the greatest benefit. Other benefits reported included reducing therapist travel time $(9.5 \%, n=23)$, professional growth $(7.5 \%, n=14)$, greater opportunity for collaboration $(2 \%, n=2)$, support for generalisation and carryover $(2 \%, n=2)$ and that telepractice can be a monitoring support for face-to-face therapy (1\%, $n=1)$.
Table 3

Barriers to using telepractice

\begin{tabular}{lc}
\hline Barrier & $\begin{array}{c}\text { Percentage of } \\
\text { Respondents }\end{array}$ \\
\hline Therapy more suited to face to face & $34.5 \%$ \\
Technology concerns & $24.5 \%$ \\
Client preference for face-to-face therapy & $12 \%$ \\
Organisation barriers & $11.5 \%$ \\
Building rapport with client & $7 \%$ \\
Lack of training \& guidance & $7 \%$ \\
Lack of evidence base & $6 \%$ \\
Therapist reluctance to use telepractice & $0.5 \%$ \\
Limits multidisciplinary collaboration & $0.5 \%$ \\
\hline
\end{tabular}

\subsection{Barriers impacting telepractice use}

Respondents were surveyed about their perceived barriers to using telepractice (see Table 3). Over a third of respondents $(34 \%, n=30)$ reported that some therapeutic activities were more suitable for face-to-face therapy and not amenable to telepractice (with reasons related to the client profile or type of therapy given). One participant commented that challenges arose if the client had difficulty navigating the technology needed to implement telepractice sessions: "One must be cognizant of the person's level of technological/IT literacy... These clients cannot be unfairly disadvantaged in comparison to those with IT literacy".

Technology concerns were also a considerable barrier, with almost one quarter reporting challenges with equipment, connectivity and sound quality. 
Participants made reference to poor ICT infrastructure in rural communities and how certain telepractice platforms were unsuitable for particular aspects of therapy. For example, one respondent explained that prolonged vowels were picked up as background noise and muted on some video-conferencing platforms making voice therapy sessions difficult.

Organisational barriers including policy and procedure challenges were reported by $12 \%(n=11)$. Client's preference for face-to-face therapy $(12 \%$, $n=11$ ) and difficulties building rapport were further barriers reported. One respondent indicated that telepractice may not offer the same opportunities to engage with families: "While attending for SLT is merely a small part of a child's journey in life, you are working with children and parents in potentially very vulnerable positions, and having a strong, positive relationship is vital towards achieving the most positive outcome for that child. I think this can only be truly achieved through face-to-face". A small number of respondents identified a lack of training and guidance $(7 \%, n=6)$ and evidence base $(6 \%, n=5)$ as barriers to telepractice use. Finally, one respondent reported their own personal reluctance to using telepractice $(0.5 \%, n=1)$ and another felt it did not support multidisciplinary collaboration $(0.5 \%, n=1)$.

\subsection{Facilitators of telepractice use}

The majority of respondents $(92 \%, n=159)$ identified the support of communication partners as a key facilitator of telepractice delivery. Almost two thirds of respondents $(65 \%, n=112)$ relied on assistance from the parent or caregiver and 20\% depended on the clients' spouse or partner $(n=35)$. "Other" helpers were selected by $15 \%$ of participants, who referenced the clients' children and grandchildren $(n=7)$ and staff in residential settings $(n=5)$. Respondents were asked how the communication partner helps in telepractice sessions and most communication partners were reported to assist with technology (34\%, $n=59)$ (see Fig. 4). Over one fifth $(22 \%, n=38)$ mentioned practising newly learned behaviours, $18 \%$ $(n=31)$ said assisting with assessment and $17 \%$ $(n=29)$ answered homework. Several participants wrote that the communication partner helped by providing verbal feedback to the SLT on what the client was doing or supporting the client to remain engaged in the session.

Participants also recognised that using telepractice to deliver SLT required new skills and knowledge. More than half the participants were self-taught
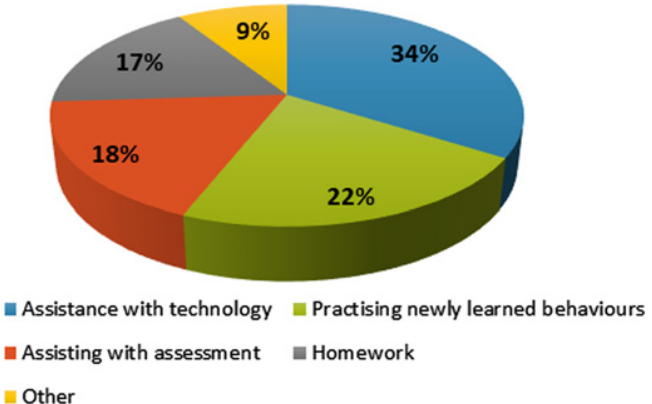

Fig. 4. How a communication partner assists in a telepractice session.

through personal experience $(53 \%, n=47) .13 \%$ were informally trained by colleagues $(n=12)$, with just $6 \%$ receiving formal training $(n=5)$. For those who selected the "other" option $(28 \%, n=25)$, practitioners mentioned telepractice webinars, consulting IASLT (2020), RCSLT (2020) and ASHA (2020) telepractice guidelines and problem solving with colleagues.

Participants were asked to identify the supports that would strengthen their delivery of telepractice. Technology upgrades was selected by over a quarter of respondents $(28 \%, n=48)$. Supports to develop knowledge and skill were identified by many respondents including training courses on telepractice use for different clinical populations and different types of therapy, for example group work $(27 \%, n=47)$, discussion boards to share their experiences with telepractice in Ireland $(25 \%, n=43)$ and increased access to telepractice literature and webinars $(17 \%$, $n=29$ ). ICT support and leadership from management were identified as important potential supports for $4 \%(n=7)$ of respondents. Finally, $90 \%(n=156)$ recognised that education on the topic of telepractice should be included on all undergraduate and postgraduate university courses to ensure that future SLTs are prepared for using telepractice.

\section{Discussion}

This anonymous online survey documents a seismic shift in the delivery of SLT services in Ireland. Following the onset of the COVID-19 pandemic, $51 \%$ of respondents from this representative sample of SLTs registered in Ireland turned to telepractice as a means of continuing SLT services during lockdown. Prior to the pandemic, a survey of health professionals in Ireland indicated that only $3 \%$ were 
using telepractice (HSCP, 2020), highlighting the scale of this rapid change, mirroring findings from international research reporting the acceleration of telepractice in 2020 (Dimer et al., 2020; Mac Evilly \& Brosnan, 2020; Tohidast et al., 2020). The COVID-19 pandemic has acted as a radical disruptor to all aspects of society. While it has brought many severe difficulties and challenges, the rapid progress in telepractice adoption is likely to be beneficial for clients and services in the long term due to its documented benefits (Morris et al., 2019; Swales et al., 2019). It addresses key strategic priorities (e.g., Slaintecare, 2019; WHO, 2020) and has the potential to enhance SLT delivery in Ireland.

As a key service delivery option during severe public health restrictions, telepractice was implemented wherever possible, which gave SLTs the opportunity to evaluate how telepractice might best be utilised in a post-COVID context. Respondents recognised that telepractice is not a replacement for face-to-face therapy, but a valuable adjunct for particular client groups and for specific aspects of therapy. These findings suggest telepractice is best utilised alongside faceto-face therapy, as part of a blended model of service delivery differentiated by client needs.

Aside from being able to continue services in the context of public health restrictions, a core benefit of telepractice reported by respondents was the ability to work with clients in their own environments. SLTs have long been concerned with supporting the participation of individuals with speech, language, communication and swallowing difficulties (Grillo, 2017; Nordio et al., 2018; WHO, 2001). The challenges of translating therapeutic gains made in the clinic room to the real world have been an ongoing challenge for therapists (RCSLT, 2020). Telepractice may offer a bridge to supporting communicative participation without the costs associated with home visits. Even for clients who may not be best served by telepractice as their main service delivery model, telepractice could offer insights and observational opportunities to inform and maximise the effectiveness and individualisation of therapy.

A major barrier to telepractice use was that it did not suit all clients (particularly those with more complex profiles) or all types of therapy (for example, dysphagia intervention). This survey highlights the need for decision making resources to support SLTs in choosing when to use telepractice and when face-to-face may be more appropriate. A further considerable barrier identified was technology related. Many respondents reported challenges with technology infrastructure such as broadband connectivity or access to ICT equipment. These findings lend further weight to the urgent need for the rapid rollout of the National Broadband Plan (Department of the Environment, Climate \& Communications, 2021) to ensure widespread access to high quality internet connectivity, particularly for those in rural contexts who may also have further to travel for face-to-face services. A smaller group reported challenges related to audio quality, a particular concern for a profession assessing speech and voice, suggesting a need for specific technological solutions for these aspects of therapy (Weerathunge et al., 2021).

The survey suggests that half of respondents adopted telepractice during COVID-19. However, further change and support is clearly needed to embed telepractice universally within SLT. The facilitators of telepractice identified in this study may offer guidance to support further telepractice adoption. Respondents valued the support of communication partners in delivering therapy remotely. Communication partners took on a variety of roles and their active involvement was seen as an advantage over face-toface therapy. The need for communication partners who can support and mediate telepractice sessions was widely recognised in the literature (Law et al., 2021). Preparation and success of telepractice may be supported by the timely identification of potential communication partners and by providing concrete support for them in their role.

Respondents also identified a number of easy to implement supports that would further enhance telepractice implementation. They valued opportunities to learn from colleagues and proposed the establishment of support networks that may provide opportunities for SLTs to share their telepractice experiences. There was also a recommendation for more formal instruction and support to implement telepractice, including access to peer-reviewed literature, availability of expert-led webinars and participation in professional development courses. These suggestions are echoed in Ireland's national eHealth Strategy (Department of Health, 2020) which identified integrative collaboration and appropriate staff training as "fundamental enablers" to the successful implementation of telepractice.

Amalgamating the findings of this study with existing frameworks of change may support improved implementation of telepractice innovations within SLT and enhanced evaluation of telepractice as an adjunct SLT delivery model. This may be achieved through supporting a more holistic consideration 


\section{Essential conditions}

$\checkmark$ Are you capable of implementing telepractice physically and psychologically? (e.g., Have you received training or coaching? Do you have the necessary ICT equipment?)

$\checkmark$ Do you have the opportunity to implement telepractice physically and socially? (e.g., Are there options to adapt your current service pathways to include telepractice? Would telepractice be suitable for your client group and their current communication and/or swallowing difficulties?)

$\checkmark$ Are you motivated to implement telepractice? (e.g., Self- motivated or externally motivated?)

\section{Intervention strategies to support the above essential conditions}

Can you avail of:

$\checkmark$ education (e.g., access to relevant literature)

$\checkmark$ incentivisation (e.g., CPD credits)

$\checkmark$ training (e.g., formal/informal/taught/self-directed/webinars)

$\checkmark$ environmental restructuring (e.g., office space, telepractice background)

$\checkmark$ modelling (e.g., observing a colleague)

$\checkmark$ peer mentoring and support (e.g., journal club, troubleshooting)

$\checkmark$ support from a communication partner (e.g., parent, family member)

\section{Policy and contextual supports}

Can you access:

$\checkmark$ support network and discussion groups about telepractice in SLT and other services (e.g., Special Interest Group)

$\checkmark$ telepractice guidelines (e.g., national and international guidelines; IASLT, HSE, ASHA, RCSLT, Speech Pathology Australia)

$\checkmark$ financial support (e.g., purchase of necessary ICT resources)

$\checkmark$ professional regulation and policy guidance for telepractice (e.g., professional body clinical guidelines)

$\checkmark$ supportive legislation (e.g., GDPR legislative framework for sharing data online)

Fig. 5. Proposed checklist for SLTs considering introducing telepractice (adapted from the Behavioural Change Wheel (Michie et al., 2011) and the findings of this study).

of (a) the individual implementing telepractice, (b) strategies and practices to implement telepractice, and (c) systemic and contextual factors. For example, the Behavioural Change Wheel (Michie et al., 2011) has multiple levels that could be applied to telepractice implementation in SLT at a local level including its (1) three essential conditions: capability - both physical and psychological capability; opportunity - both physical and social opportunity; and motivation - both automatic processes and reflective processes; (2) intervention functions to address any challenges (e.g., education, training, modelling) and (3) policy categories (e.g., regulation, legislation). This focus on broader contextual aspects of change, as well as the individual clinician expected to implement telepractice, may provide further direction for embedding effective and client-centred telepractice into SLT service delivery. See Fig. 5 for a suggested 
facilitative checklist for SLTs and SLT managers considering implementing telepractice into their service delivery model, based on the findings of this study and the Behavioural Change Wheel (Michie et al., 2011).

\section{Limitations}

The generalisability of the results are subject to certain limitations. For instance, participants were recruited through Twitter or email. This recruitment method may have increased the potential bias of the sample and the likelihood that participants were proficient with technology, which is associated with enhanced clinician acceptance of telehealth (HSCP, 2020). While such convenience and snowball sampling may have influenced the respondents that the present survey could reach (Bryman, 2012), the sample of clinicians who participated represents a range of staff grades, experience, work settings, and those who work with a diverse range of client groups.

Reports of client feedback on their experiences of telepractice must be interpreted with caution, as this study did not collect data on perspectives of service users directly. Further research should focus on clients' experiences of telehealth in SLT (HSCP, 2020) and seek to triangulate data with other key stakeholders' perspectives such as service managers or multi-disciplinary colleagues. Follow on focus groups may have enabled key findings to be discussed in more detail, providing greater context and opportunities to elaborate on facilitators of telepractice and suggest means of addressing barriers identified.

The timing of this survey coincided with significant and sudden disruptions in SLT services in Ireland in response to the first wave of COVID-19 and public health guidance of reducing face-to-face contacts in healthcare delivery where possible. As the survey was cross-sectional, it depicts telepractice use in Ireland during a specific timeframe in the earlier period of the COVID-19 pandemic (June-September 2020) and telepractice implementation has possibly evolved considerably since the survey was conducted.

\section{Future directions}

Concerns regarding efficacy have long hampered telepractice uptake (Smith et al., 2020; Swales et al., 2019; Zughni et al., 2020). In the current study, several respondents were apprehensive about telepractice use with their caseload or specific modes of intervention (e.g., clients with disabilities or delivering group sessions). Further research is required to establish telepractice efficacy with a wider range of client groups and types of intervention to ensure evidence-based and client-centred care. This will include exploration of service users' opinions to identify their perceived benefits and barriers of receiving SLT via telepractice and address any challenges identified. This study has also proposed a facilitative checklist for SLTs who are considering introducing telepractice in their service. We hope to evaluate the usefulness and applicability of the checklist, identifying possible additions or adjustments required. In addition, we aim to investigate which facilitative factors identified in the checklist have the most impact on adoption of telepractice (e.g., ICT infrastructure, professional development etc.) and whether gaps exist in SLTs' ability to access facilitative factors. Furthermore, we plan on evaluating the impact and effectiveness of education and preparation of undergraduate students for the use of telepractice in their future careers in SLT.

\section{Conclusion}

This study highlights the rapid increase in telepractice uptake by the SLT profession in Ireland during COVID-19 to support service continuity in the midst of wide-ranging public health restrictions. It identifies benefits for clinicians and clients, in addition to barriers and facilitators for the future use of telepractice as part of a blended SLT service delivery model. A facilitative checklist for SLTs considering implementing telepractice is proposed based on the study findings and an existing model of behavioural change.

\section{Acknowledgments}

The authors wish to thank the SLTs who responded to the survey and shared their perspectives on telepractice.

No funding was received for this study.

\section{Author contributions}

EF and YL conceived the study and defined the study aims. EF devised the survey, collected the data and conducted the data preparation and statistical 
analysis. All authors contributed to data analysis, interpretation of findings, preparation and approval of the final manuscript.

\section{Conflict of interest}

The authors have no conflict of interest to report.

\section{Ethical considerations}

This study protocol was reviewed and approved by the School of Linguistic, Speech and Communication Sciences Research Ethics Committee Trinity College Dublin, approval number TT38.

Consent to participate statement: Written informed consent was not required as the survey was anonymous and collected no personal data. Potential participants were provided with information about the study and then pressed a button in the survey indicating they consented before proceeding to the survey items.

\section{Supplementary material}

The appendix is available in the electronic version of this article: https://dx.doi.org/10.3233/ACS210036.

\section{References}

Akamoglu, Y., Meadan, H., Pearson, J., \& Cummings, K. (2018). Getting connected: speech and language pathologists' perceptions of building rapport via telepractice. Journal of Developmental and Physical Disabilities, 30(4), 569-585. https://doi. org/10.1007/s10882-018-9603-3

AHA. (2021). Telehealth: Advancing health in America. American Hospital Association. Retrieved 20 January 2021, from https:// www.aha.org/telehealth

ASHA. (2016). Special interest group 18: Telepractice survey results. American Speech-Language-Hearing Association. Retrieved 23 September 2020, from https://www.asha.org/upload edFiles/ASHA/PracticePortal/ProfessionalIssues/Telepractice/ 2016-Telepractice-Survey.pdf

ASHA. (2021). Providing telehealth services under Medicare during the COVID-19 pandemic. American Speech-LanguageHearing Association. Retrieved 5 October 2020, from https:// www.asha.org/Practice/reimbursement/medicare/ProvidingTelehealth-ServicesUnder-Medicare-During-the-COVID-19Pandemic/

Bryman, A. (2012). Social research methods (4th ed.). London: Oxford University Press.
CORU. (2020). CORU registration statistics. Retrieved 20 July 2021, from https://www.coru.ie/news/news-for-the-public/ coru-registration-statistics-november-2020.html

Department of Health. (2020). eHealth Strategy for Ireland. Health Service Executive. Retrieved 3 August 2021, from https:// www.ehealthireland.ie/knowledge-information-plan/ehealthstrategy-for-ireland.pdf

Department of Health. (2019). Sláintecare Action Plan. Health Service Executive. Retrieved 18 October 2020, from https:// assets.gov.ie/9379/05384619bb2240c18c294b60578117e1. pdf

Department of the Environment, Climate and Communications. (2021). National broadband plan. Gov.ie. Retrieved 13 August 2021, from https://www.gov.ie/en/publication/c1b0c9-nation al-broadband-plan/

Dimer, N., Canto-Soares, N., Santos-Teixeira, L., \& Goulart, B. (2020). The COVID-19 pandemic and the implementation of telehealth in speech-language and hearing therapy for patients at home: An experience report. CoDAS, 32(3), e20200144. https://doi.org/10.1590/2317-1782/20192020144

Doll, E., Braden, M., \& Thibeault, S. (2021). COVID-19 and speech-language pathology clinical practice of voice and upper airway disorders. American Journal of Speech-Language Pathology, 30(1), 63-74. https://doi.org/10.1044/2020_ajslp20-00228

Fong, R., Tsai, C., \& Yiu, O. (2020). The implementation of telepractice in speech language pathology in Hong Kong during the COVID-19 pandemic. Telemedicine and eHealth, 27 (1), 30-38. https://doi.org/10.1089/tmj.2020.0223

Greenhalgh, T., Wherton, J., Shaw, S., \& Morrison, C. (2020). Video consultations for COVID-19. BMJ, 63(9), 368-371. https://doi.org/10.1136/bmj.m998

Grillo, E. (2017). Results of a survey offering clinical insights into speech-language pathology telepractice methods. International Journal of Telerehabilitation, 9(2), 25-30. https:// doi.org/10.5195/ijt.2017.6230

Health and Social Care Professions (2020). Telehealth: What matters to you? Galway: Health and Social Care Professions Office.

Hernandez, K., \& Roberts, T. (2018). Leaving no one behind in a digital world. K4D Emerging Issues Report. Retrieved 5 November 2020, from https://assets.publishing.service.gov. uk/media/5c178371ed915d0b8a31a404/Emerging_Issues_LN OBDW_final.pdf

Hill, A., \& Miller, L. (2012). A survey of the clinical use of telehealth in speech-language pathology across Australia. Journal of Clinical Practice in Speech Lang Pathology, 14 (3), 110-117.

IASLT. (2020). COVID-19: Guidance for IASLT Members. Irish Association of Speech and Language Therapists. Retrieved 4 October 2020, from https://www.iaslt.ie/documents/public information/COVID\%2019\%20IASLT\%20Guidelines/IASLT \%20COVID-19\%20Guidance\%20March2020.pdf

IBM Corporation. (2019). IBM SPSS Statistics for Windows, Version 26.0. Armonk, NY: IBM Corporation.

Kuva Kraljevi, J., Mati, A., \& Pavil Dokoza, K. (2020). Telepractice as a reaction to the COVID-19 crisis: Insights from Croatian SLP settings. International Journal of Telerehabilitation, 12(2), 93-104. https://doi.org/10.5195/ijt.2020.6325

Law, J., Dornstauder, M., Charlton, J., \& Gréaux, M. (2021). Tele-practice for children and young people with communication disabilities: Employing the COM-B model to review the intervention literature and inform guidance for practition- 
ers. International Journal Of Language \& Communication Disorders, 56(2), 415-434.https://doi.org/10.1111/1460-6984. 12592

MacEvilly, D., \& Brosnan, G. (2020). Adapting an emotional regulation and social communication skills group programme to teletherapy, in response to the COVID-19 pandemic. Irish Journal of Psychological Medicine, 1(6), 1-6. https://doi.org/ 10.1017/ipm.2020.109

Michie, S., van Stralen, M.M. \& West, R. (2011). The behaviour change wheel: A new method for characterising and designing behaviour change interventions. Implementation Science, 6(42), 32-50. https://doi.org/10.1186/1748-5908-6-42

Mohan, H., Anjum, A., \& Rao, P. (2017). A survey of telepractice in speech-language pathology and audiology in India. International Journal of Telerehabilitation, 9(2), 69-80. https:// doi.org/10.5195/ijt.2017.6233

Morris, J., Jones, M., Thompson, N., Wallace, T., \& DeRuyter, F. (2019). Clinician perspectives on mrehab interventions and technologies for people with disabilities in the United States: A national survey. International Journal of Environmental Research and Public Health, 16(21), 20-42. https://doi.org/ 10.3390/ijerph16214220

Nordio, S., Innocenti, T., Agostini, M., Meneghello, F., \& Battel, I. (2018). The efficacy of telerehabilitation in dysphagic patients: a systematic review. Acta otorhinolaryngologica Italica, 38(2), 79-85. https://doi.org/10.14639/0392-100X-1816

Pennington, L., Stamp, E., Smith, J., Kelly, H., Parker, N., \& Stockwell, K. et al. (2019). Internet delivery of intensive speech and language therapy for children with cerebral palsy: A pilot randomised controlled trial. $B M J, 9(1), 24-33$. https://doi.org/ 10.1136/bmjopen-2018-024233

Qualtrics. (2021). Sample Size Calculator. Qualtrics XM. Retrieved 9 August 2020, from https://www.qualtrics.com/blog/ calculating-sample-size/

Qualtrics Provo. (2005). Qualtrics Version 6.20. Qualtrics XM. Retrieved 9 June 2020, from https://www.qualtrics.com

RCSLT. (2020). Telehealth Guidance. Royal College of Speech and Language Therapists. Retrieved 4 October 2020, from https://www.rcslt.org/members/deliveringquality-services/ telehealth/telehealth-guidance

Sharma, A., Minh Duc, N., Luu Lam Thang, T. et al. (2021). A consensus-based checklist for reporting of survey studies (CROSS). Journal of General Internal Medicine. https:// doi.org/10.1007/s11606-021-06737-1

Smith, A., Thomas, E., Snoswell, C., Haydon, H., Mehrotra, A., Clemensen, J., \& Caffery, L. (2020). Telehealth for global emergencies: Implications for coronavirus disease 2019 (COVID-19). Journal of Telemedicine and Telecare, 26(5), 309-313. https://doi.org/10.1177/1357633x20916567
Swales, M., Theodoros, D., Hill, A., \& Russell, T. (2019). Speechlanguage pathologists' perceptions of the use of telepractice in the delivery of services to people with Parkinson's disease: A national pilot survey. International Journal of SpeechLanguage Pathology, 22(4), 387-398. https://doi.org/10.1080/ 17549507.2019.1650110

Tohidast, S., Mansuri, B., Bagheri, R., \& Azimi, H. (2020). Provision of speech-language pathology services for the treatment of speech and language disorders in children during the COVID19 pandemic: Problems, concerns, and solutions. International Journal of Paediatric Otorhinolaryngology, 13(8), 11-26. https://doi.org/10.1016/j.ijporl.2020.110262

Tucker, J. (2012). Perspectives of speech-language pathologists on the use of telepractice in schools: Quantitative survey results. International Journal of Telerehabilitation, 4(2), 6-13 https:// doi.org/10.5195/ijt.2012.6100

Wales, D., Skinner, L., \& Hayman, M. (2017). The efficacy of telehealth-delivered speech and language intervention for primary school-age children: A systematic review. International Journal of Telerehabilitation, 9(1), 55-70. https://doi.org/ 10.5195/ijt.2017.6219

Weerathunge, H., Segina, R., Tracy, L., \& Stepp, C. (2021). Accuracy of acoustic measures of voice via telepractice videoconferencing platforms. Journal Of Speech, Language, and Hearing Research, 64(7), 2586-2599. https://doi.org/10.1044/ 2021_jslhr-20-00625

Weidner, K., \& Lowman, J. (2020). Telepractice for adult speech-language pathology services: A systematic review. Perspectives of The ASHA Special Interest Groups, 5(1), 326-338. https://doi.org/10.1044/2019_persp-19-00146

World Health Organisation. (2001). International classification of functioning, disability and health: ICF. World Health Organisation. Retrieved 5 December 2020, from https://apps.who.int/ iris/handle/10665/42407

World Health Organisation. (2020). Global strategy on digital health 2020-2025. World Health Organisation. Retrieved 6 December 2020, from https://www.who.int/docs/default source/documents/gs4dhdaa2a9f352b0445bafbc79ca799dce 4d.pdf

Zughni, L., Gillespie, A., Hatcher, J., Rubin, A. \& Giliberto, J. (2020). Telemedicine and the Interdisciplinary clinic model: During the COVID-19 pandemic and beyond. Otolaryngology-Head and Neck Surgery, 163(4), 67-75. https://doi.org/ $10.1177 \% 2 \mathrm{~F} 0194599820932167$ 